\title{
Stable families of coalitions for network resource allocation problems
}

\author{
Vladimir Gurvich ${ }^{1}$ and Sergei Schreider ${ }^{2}, *$ \\ ${ }^{1}$ RUTCOR, Rutgers Center for Operations Research, Rutgers University, USA \\ 2 School of Mathematical and Geospatial Sciences, RMIT University, Australia
}

Received 15 Octber 2013; Accepted 18 December 2013

Editor: Vassili Kolokoltsov

\begin{abstract}
A very common question appearing in resource management is: what is the optimal way of behaviour of the agents and distribution of limited resources. This paper addresses the following question: Is there at least one form of cooperation that satisfies interests of all players better than competition? This research is based on results proving the existence of a non-empty $\mathcal{K}$-core, that is, the set of allocations acceptable for the family $\mathcal{K}$ of all feasible coalitions, for the case when this family is a set of subtrees of a tree.

A wide range of real situations in resource management, which include optimal water, gas and electricity allocation problems, can be modeled using this class of games, whose $\mathcal{K}$-core is non-empty due to the acyclic structure of the associated networks. Thus, the present research is pursuing two goals: 1 . optimality and 2 . stability.

Firstly, we suggest to players to unify their resources and then we optimize the total payoff using some standard LP technique. The same unification and optimization can be done for any coalition of players, not only for the total one. However, players may object unification of resources. It may happen when a feasible coalition can guarantee a better result for every coalitionist. Here we obtain some stability conditions which ensure that this cannot happen for some family $\mathcal{K}$. Such families were characterized by Boros, Gurvich and Vasin [4] as Berge's normal hypergraphs. Thus, we obtain a solution which is optimal and stable. From practical point of view, we suggest a distribution of profit that would cause no conflict between players.
\end{abstract}

Keywords Game theory, network system, normal hypergraph, GBV Theorem

DOI: $10.19139 /$ soic.v2i1.28

'What to suggest? .... They write and write [....]. It makes my head reel. Just take everything and share equally...' - Michail Bulgakov The heart of a dog

\footnotetext{
${ }^{*}$ Correspondence to: School of Mathematical and Geospatial Sciences, RMIT University, GPO Box 2476, Melbourne Vic 3001, Australia, email: Sergei.Schreider@rmit.edu.au
}

ISSN 2310-5070 (online) ISSN 2311-004X (print)

Copyright (c) 2014 International Academic Press 


\section{Introduction}

\subsection{Rationale}

Optimal performance of multi-agent economic systems is a pivotal problem of the modern economics. Performance quality of such systems can be described by a set of numerical characteristics reflecting the way these systems operate. These characteristics could include profit, cost, gross margin, quantity or number of produced commodities, and will be referred to henceforth as Objective Functions (OF). The primal managerial task is to optimize (maximize or minimize) these OF. Without loosing the generality, the maximization formulation will be considered.

There are two possible approaches to selecting the optimal strategy. The first one (henceforth 'collective' approach) is used when an overall system performance is considered and individual agents' performances are not the main focus of the research. In this case, the most common tool employed for the system optimization is the non-linear or linear programming, usually integer or mixed. The second approach implies optimization considered from each of the agents' point of view (henceforth referred to as 'individual'). With this approach, the system optimization tool is the game theoretic methods. The fist approach (overall optimization) is equivalent to the second approach (game) when all agents form one large coalition. The value of overall maximized OF is not less than the sums of the individual OFs obtained by individual players or coalitions as solutions of a game. It is tempting to suggest that agents form one coalition and then divide the total payoff between them. However, some players (or coalitions of players) might be better off pursuing strategies different from the strategy formulated for the overall optimization for 'one large coalition' game. Moreover, players or coalitions of players, which are better off playing separately, exist in the general case.

This paper describes quite a wide range of systems for which the non-conflicting distribution of optimal 'collective' OFs between a system's agents is possible. These systems belong to the class of the so-called network systems and are characterized by the existence of carriers connecting individual players who are located in nodes. The performance of such systems can be optimized using the network LP methods, containing a set of very specific constraints, related to the capacity of the carriers and the existence of paths between different nodes. A wide range of real economic systems (water supply networks, electricity and gas supply networks, telecommunications, etc.) can be described as such network systems, which makes the results of the reported research highly demanded by managerial practices in these areas. The fundamental result employed in this paper states that for such systems there exists a non-conflicting redistribution of total income between the players. 


\subsection{Mathematical formulation}

We consider the situation when a group of agents $I$ that has an access to the set of limited resources $R$ has a goal to optimize some objective function. $P$ is the set of products (industries). We ask the following question: what optimal cooperation strategy, or coalition structure, should be chosen by the agents in order to optimize their economic outputs.

The linear case appears when for each player $i \in I$ his strategies $x_{i}$ are the shares of his activities in industry (product) $p \in P$. Let

$$
A=\left\|a_{r p}\right\|
$$

be a matrix representing the amount of resource $r \in R$ needed for producing a unit of product $p \in P$, and

$$
b^{i}=\left(b_{1}^{i}, b_{2}^{i}, \ldots, b_{|R|}^{i}\right)
$$

be a vector of resources available for player $i$.

Then we can formulate the production strategy choice for each player as a LP problem:

Maximize the revenue function

$$
f(x)=\sum_{p \in P} c_{p} x_{p}
$$

Subject to $A x \leq b, x \geq 0$, where $b$ is a vector of available resources and $c_{p}$ are the prices per unit of products $p$.

This formulation can be settled for each player $i \in I$ or coalition $K \subseteq I$.

Respectively, we replace $b$ by $b^{i}$ or $b^{K}$. For each coalition $K$ vector $b^{K}$ is additively defined:

$$
b^{K}=\sum_{i \in K} b^{i}
$$

The corresponding LP solution will be denoted as $x^{*}$; the optimal solution is a vector

$$
x^{*}=\left(x_{i}^{*}, i \in I\right)
$$

Let us define function $v$ on the set of coalitions as:

$$
v(K)=\sum_{i \in K} x_{i}^{*}\left(b^{K}\right)
$$

This function is superadditive, that is, for each two disjoint coalitions $K^{\prime}$ and $K^{\prime \prime}$

$$
v\left(K^{\prime} \cup K^{\prime \prime}\right) \geq v\left(K^{\prime}\right)+v\left(K^{\prime \prime}\right) \forall K^{\prime} K^{\prime \prime} \subseteq I \text { such that } K^{\prime} \cap K^{\prime \prime}=\emptyset .
$$

This result was proven by Johnson [16], Gomory and Johnson [9] and further developed by Blair and Jeroslow [3], Jeroslow [14, 15] and Schrijver [20]. It is 
referred in the literature as superadditive (or subadditive) duality. We can combine it with BGV theorem (see paper of Boros, Gurvich and Vasin [4, 5] and the originating papers of Gurvich and Vasin $[10,11])$ stating that family of coalitions

$\mathcal{K} \subseteq 2^{I}$ is stable (that is the $\mathcal{K}$-core $C(v, \mathcal{K})$ is not empty for any superadditive characteristic function $v$ ) if and only if $\mathcal{K}$ is a normal hypergraph, according to Berge [1].

As an application, we plan to consider an example of particular game, in which the corresponding graph is a water allocation system represented by a network of gravitationally driven water carriers (rivers, canals, pipelines). We show that if a coalition is created by the neighbour players (farmers) whose properties are located along the same water supply carrier, or along a tree formed by such carriers, then the core of the obtained cooperative game is not empty. Let us now define accurately the concepts considered above.

\section{Stable families of coalitions, normal hypergraph and TU-games}

For the beginning, let us consider cooperative games with transferable utility (TUgames).

The notation is formalized as follows: Let $I$ be a set of players. Its subsets $K \subseteq I$ are called coalitions. A TU game is defined by a characteristic function $v: 2^{I} \rightarrow R$.

Vector $\left(\mathrm{x}_{i}: i \in I\right)$ is called an allocation if $\sum_{i \in I} x_{i} \leq v(I)$. Here $x_{i}$ is interpreted as a payoff of player $i \in I$.

Furthermore,

$$
x_{K}=\sum_{i \in K} x_{i}
$$

is a payoff of coalition $K \subseteq I$.

The core $C(v)$ of the obtained superadditive TU game is defined by the system of linear inequalities

$$
C(v)=\left\{x \in R^{|I|} \mid x_{I} \leq v(I) \text { and } x_{K} \geq v(K) \forall K \subseteq I\right\}
$$

It can be interpreted as a set of allocations $\left(\mathrm{x}_{i}: i \in I\right)$ acceptable for all coalitions

$$
K \subseteq I
$$

In other words, the core of a TU-game is empty whenever each allocation is rejected by a coalition. The core is a natural and probably the simplest concept of solution in cooperative game theory. Yet it has an important disadvantage: $C(v)$ is frequently empty, because it must be acceptable for all $2^{|I|}$ coalitions.

Yet in real life not every coalition has a chance to appear, because some agents may not know or not like each other. The following relaxation of the concept is common in the literature: 
Given a family of coalitions $\mathcal{K} \subseteq 2^{I}$ the $\mathcal{K}$-core is defined as a family of allocations acceptable for all coalitions $K \in \mathcal{K}$ that is

$$
C(v, \mathcal{K})=\left\{x \in R^{|I|} \mid x_{I} \leq v(I) \text { and } x_{K} \geq v(K) \forall K \in \mathcal{K}\right\}
$$

Family $\mathcal{K} \subseteq 2^{I}$ is called stable if the $\mathcal{K}$-core is not empty for any superadditive TU-game $v: 2^{I} \rightarrow R$.

An important result obtained by Boros, Gurvich and Vasin [4], which is referred to as the $\mathrm{BGV}$ theorem, claims that family of coalitions $\mathcal{K}$ is stable if and only if $\mathcal{K}$ is a Berge normal hypergraph (see his works $[1,2])$. The definitions of terms 'perfect graph', 'normal hypergraph' and explanation of links between games, coalitions and hypergraphs are given in Appendix to Gurvich and Schreider [12], accessible online (http: //rutcor.rutgers.edu/pub/rrr/reports2010/20_2010.pdf).

For the present paper the following example is most important: Let $T$ be a tree, assign a player to every vertex of $T$. Then an arbitrary family of subtrees of $T$ forms a stable family of coalitions.

This special case of the BGV theorem, considered already in Gurvich and Vasin [4], is important in applications. As it has been already mentioned above, we consider the family of LP problems whose resource constraints (right-hand side) $b^{K}$ depends on a coalition. Hence, the optimal solution

$$
v(K)=\sum_{i \in K} x_{i}^{*}\left(b^{K}\right)=x^{*}(K)
$$

is a superadditive function of $K$. Thus the BGV theorem is applicable. Hence, it exist such a distribution of the total optimal payoff $v(I)$ among the players that will cause no objection from any coalition.

\section{Network supply systems and games}

Water, electricity and gas supply systems fall into the class of the so-called network systems. The common characteristics of all these allocation systems is that they can be represented as a set of nodes (for instance, reservoirs, junctions and users) interconnected by a system of carriers with different capacities (wires, channels, pipelines). The last two decades a number of publications appeared when these types of systems were described by linear and non-linear optimization methods. For water supply system this approach was employed for instance in the paper of Perera et al. [17]. Electricity supply systems and associated electricity market, as well as traffic and telecommunication systems, were considered by $\mathrm{Hu}$ and Ralph [13] and Ralph [18]. Gas supply system for Europe and North America were also modelled by similar methods of network LP optimization (see paper of Egging et al. [7] and Gabriel et al. [8]).

In order to be more specific, the REALM (Resource Allocation Model) illustrates how this network LP works for the irrigation water supply systems. More detailed 
description of this LP network optimization can be found in paper of Dixon et al. [6]. This formulation illustrates what types of the LP formulations for water allocation can be included in the framework formulated above.

Following formulation proposed by Dixon et al. [6] in stylized, form REALM models can be represented as:

Choose non-negative values for

$F(i, r, t)$, for $i \in D, r \in D \cup E, t=1,2, \ldots, T$,

$S(e, t)$, for $e \in E, t=1,2, \ldots, T$ and

$W(i, t)$ for $i \in D, t=1,2, \ldots, T$

to minimize

$$
\begin{aligned}
& \sum_{i \in D} \sum_{r \in D \cup E} \sum_{t} c_{i, r}(t) * F(i, r, t)+ \\
& \sum_{e \in E} \sum_{t} \beta_{e}(t) *|d(e, t)-S(e, t)|+ \\
& \sum_{i \in D} \sum_{t} g_{i, t}\left[W(i, t)-W_{\min }(i, t)\right]
\end{aligned}
$$

subject to

$$
\begin{aligned}
& W(i, t+1) \leq W(i, t)-\sum_{r \in D \cup E} F(i, r, t)+ \\
& \sum_{k \in D} F(k, i, t) *[1-1(k, i, t)]+ \\
& X(i, t)-\theta_{i, t}[W(i, t)]
\end{aligned}
$$

for $i \in D, t=1,2, \ldots, T$

$W(i, t) \leq C(i)$ for $i \in D, t=1,2, \ldots, T$ and

$S(e, t)=\sum_{i \in D} F(i, e, t) *[1-\mathrm{l}(i, e, t)]$ for $e \in E, t=1,2, \ldots, T$

where:

$D$ is the set of dams. Dams include not only water storage facilities but also junctions in the water network. A junction has either more than one inlet or more than one outlet. It can be treated as a dam with zero capacity;

$E$ is the set of end users;

$F(i, r, t)$ is the flow in period $t$ from dam $i$ to dam or end-use $r$;

$T$ is the last period of interest. If the model were solved for one year with periods of one month, then $T=12$;

$W(i, t)$ is the amount of water in dam $i$ at the beginning of period $t . W(i, 0)$ is exogenous;

$S(e, t)$ is the amount of water supplied to end-user $e$ in period $t$;

$d(e, t)$ is the exogenously determined ideal water requirements of end-user $e$ in period $t$;

$c_{i, r}(t)$ is the cost of sending a unit of water from dam $i$ to dam or end-user $r$ in period $t$. If it is physically impossible to send water from $i$ to $r$, then $c_{i, r}(t)$ can be set at an arbitrarily large number;

$\beta_{e}(t)$ is the penalty or cost per unit of shortfall in meeting the water demands of end-user $e$ in period $t$;

$W_{\text {min }}(i, t)$ is the minimum level of water for dam $i$ that is desirable from an environmental or aesthetic point of view; 
$g_{i, t}$ is a penalty function. It takes positive values if $W(i, t)-W_{\min }(i, t)$ is negative;

$l(k, i, t)$ is losses per unit of flow from dam $k$ to dam or end-user $i$ in period $t$ (exchange losses);

$X(i, t)$, specified exogenously, is the natural inflow to dam $i$ in period $t$;

$\theta_{i, t}$ is a function giving evaporation from dam $i$ in period $t$;

$C(i)$ is the capacity of dam $i$. If dam $i$ is a junction then $C(i)=0$.

Models such as REALM can be used to plan flows in a hydrological area and to decide how these flows should be varied in response to changes in rainfall [reflected in $X(i, t)]$, changes in demands $[d(e, t)]$, and changes in a myriad of technical and cost coefficients.

As the water delivery in this system is driven by the gravity and no upstream pumping is implemented, no cycles in the water delivery graph can exist. Therefore the non-conflicting redistribution of optimal income between water users (farmers or group of farmers) does exist.

Similar optimization formulations can be used for electricity and gas supply systems.

\section{Stability of optimal solutions}

Let us consider a network LP optimization problem for an acyclic graph. Referring to the results mentioned in Section 2, we can state that a family of coalitions is stable if and only if it is a normal hypergraph. In particular any family subtrees of a tree form a stable family of coalitions. Thus, there exists an allocation corresponding to the optimal LP solution, which is stable. It means that there is a distribution of the total income among players which is acceptable for all feasible coalitions.

\section{Concluding remarks}

In nutshell, this paper suggests a new approach to optimization and fair distribution of total payoff among players. This method is based on integration of two fundamental results, which are

1. Superadditive duality for LP optimization, and

2. The BGV theorem claiming that a family of coalitions $\mathcal{K}$ is stable (that is the $\mathcal{K}$-core $C(v, \mathcal{K})$ is not empty for any superadditive characteristic function $v$ ) if and only if $\mathcal{K}$ is a normal hypergraph.

Then BGV theorem can be applied for acyclic network systems. The following two assumptions are crucial: 1. acyclicity of corresponding graph and 2 . 
superadditivity of the optimal value. It allows us to conclude that some 'natural' families of coalitions are stable, that is, admit a non-empty core. Therefore, it is possible to conclude that a fair distribution of payoff among the payers is possible. Thus, the existence of an optimal and stable solution for such class of games is proven.

A few words should be written about possible further continuation of this research. Firstly, we plan to illustrate these results by considering the real network supply system (say, the water allocation system modelled by REALM model) and demonstrate that for the full set of possible coalitions the game is unstable, that is, the core is empty. Then we will select a set of 'natural' coalitions, say, coalitions of farmers whose properties are located on the same water carrier, and demonstrate that such families are stable. The next step of this research is consideration of more sophisticated network supply systems and demonstration what are the optimal and stable solution in this case. The key point is formulation of appropriate managerial advises about redistribution of optimal income between real players constituting these systems. We also plan to consider not only TU but also more general classes of cooperative games such as games with non-transferable utilities, so-called NTU-games, also games in normal form, in effectivity function form, etc.

Here (see Section 1.2) we refer to an important result obtained by Jonson [16], Gomory and Jonson [9], Blair and Jeroslow [3], Jeroslow [15] and Schrijver [20] about superadditivity of optimal LP solutions. It should be also mentioned that the "superadditive duality" holds not only for LP but for integer programming as well. Hence, the same analyses of stability are applicable for these, more general, models.

Finally, we can consider the non-linear case related to the objective function in form of Cobb-Douglass' production function:

$$
C B\left(u_{1}, u_{2}, \ldots, u_{|R|}\right)=\prod_{i=1, \ldots, n} u_{i}^{\gamma_{i}}
$$

where $u_{i}$ are production factors and $\gamma_{1}+\gamma_{2}+\ldots+\gamma_{|P|}=1$

Schreider et al. in [19] proved that this function is also superadditive. This result gives us a potential to employ similar approach for the game on non-cyclic graphs with Cobb-Douglass objective function.

\section{Acknowledgement}

The authors would like to acknowledge Prof. Chris Cannings and two anonymous referees for their comments and suggestions on the first version of the article, which lead to significant improvements of the presentation. 


\section{REFERENCES}

1. C. Berge, Graphs and Hypergraphs, Dumond, Paris, English translation, North-Holland, Amsterdam, (1970).

2. C. Berge, Minimax theorems for normal hypergraphs and balanced hypergraphs - a survey, Annals of Discrete Mathematics, 21, (1984) pp. 3-19.

3. C.E. Blair and R.G. Jeroslow, The value function of a mixed integer program 1, Mathematical programming, 19, (1977) pp. 121-138.

4. E. Boros, V. Gurvich and A. Vasin, A stable families of coalitions and normal hypergraphs, RUTCOR Research Report 23-1995, Rutgers University; Mathematical Social Sciences 34 (1997) pp. 107-123.

5. E. Boros and V. Gurvich, Stable effectivity functions and perfect graphs, Mathematical Social Sciences, 39 (2000) pp. 175-194.

6. P. Dixon, S. Schreider and G. Wittwer, Combining engineering-based water models with a CGE model, Chapter 2 in Quantitative Tools in Microeconomic Policy Analysis, Pincus, J. (editor), Publisher: Australian Productivity Commission, Canberra (2005) pp. 17 - 30.

7. R. Egging, S.A. Gabriel, F. Holz and J. Zhuang, A complimentary model for the European natural gas market, Energy policy, 36, (2008) pp. 2385-2414.

8. S.A. Gabriel, J. Zhuang and S. Kiet A large-scale complimentary model of the North American natural gas market, Energy economics, 27, (2005) pp.639-665.

9. R.E. Gomory and E.L.,Johnson, The group problem and subadditive functions. In Hu, T.C., Robinson, S.M. (eds), Mathematical Programming, Academic Press, New York, (1973) pp. 157-184.

10. V. Gurvich and A. Vasin Reconciable sets of coalitions. In: Questions of applied math. Siberian Energetic Institute, Irkutsk, (1977) pp. 20-30 (in Russian).

11. V. Gurvich and A. Vasin Reconcilable sets of coalitions for normal form games, In "Numerical methods in optimization theory (Applied math.)", Siberian Inst. of Energy, Irkutsk, (1978) pp. 27-38 (in Russian).

12. V. Gurvich. and S. Schreider, Network supply systems, stable families of coalitions for superadditive TU-games and Berge's normal hypergraphs, RUTCOR preprint, RRR 20-2010, Rutgers University, (2010) 22 p.

13. X Hu. and D. Ralph, Using EPECs to model bilevel games inrestructured electricity markets with locational prices, Operations research, 55 (5), (2007) pp. 809-827.

14. R.G. Jeroslow, Cutting plane theory: Disjunctive methods, Annals of discrete mathematics, 1 , (1977) pp. 293-330.

15. R.G. Jeroslow, Cutting plane theory: Algebraic methods, Discrete mathematics, 23, (1978) pp. $121-150$.

16. E.L. Jonson, Cyclic groups, cutting planes and shortest paths, in Mathematical Programming, Hu, T.C. and Robinson, S. (eds.) Academic Press, (1973) pp. 185-211.

17. B.J.C. Perera, B. James and M.D.U. Kularathna, M.D.U. Computer Software Tool for Sustainable Water Allocation and Management - REALM. Journal of Environmental Management, 77 (4), (2005) pp. 291-300.

18. D. Ralph, Mathematical programs with complementarity constraints in traffic and telecommunications networks." Royal Society of London, Philosophical Transactions. Mathematical, Physical and Engineering Sciences, 366(1872), (2008) pp. 1973-1987.

19. S. Schreider, P. Zeephongsekul, B. Abbasi, and M. Fernandes, Game Theoretic Approach for Fertilizer Application: Looking for the Propensity to Cooperate, Annals of Operations Research, 206 (1), (2013) pp. 385-400.

20. A. Schrijver, On cutting planes, Annals of Discrete mathematics, 9, (1980) pp. 291-296. 